Zhang Biyun. (2020). Art on Chinese Xuan Paper. Current issues of cultural heritage in 2020. Collection of Scientific Articles. European Scientific e-Journal, 1 (1), 67-91. Hlučín-Bobrovníky: “Anisiia Tomanek" OSVČ.

DOI: $10.47451 /$ art2020-09-001

EOI: 10.11244/art2020-09-001

The paper is published in Crossref, Internet Archive, Google Scholar, Academic Resource Index ResearchBib, JGate, ISI, CiteFactor, ICI, eLibrary databases.

Zhang Biyun

Associate Professor, Candidate of Art History (PhD)

Member of the Association of Art Critics (AIS)

Member of the International Union of Teachers and Artists

College of Art, Zhejiang University of Technology

Hangzhou, Zhejiang, China

E-mail: zhangbiyun2013@163.com

\title{
Art on Chinese Xuan Paper
}

\section{Abstract:}

The formal beauty of Chinese paintings mainly lies in the peculiar material, the special brush and ink effect, the unique painting pattern, the poetry, calligraphy, painting and seal cutting, the mounting style, the composition and the technique and style of the painter. The author reveals the features of ink effect, the unique painting pattern, the poetry, calligraphy, painting and seal cutting, the mounting style, the composition and the technique and style of the painter of beauty of Chinese paintings through making contact with Chinese Xuan paper and ink because Chinese ink painting, boasting a history of more than a thousand years, represents the wisdom and talents of Chinese people. The author concludes that Chinese painting culture has a long history and boasts extensive and profound features. While it faces various problems in inheritance and dissemination in the new era.

Keywords:

Chinese paintings, Chinese Xuan paper, Chinese Xuan ink, calligraphy, seal cutting, Chinese ink painting.

\section{Introduction}

The formal beauty of Chinese paintings mainly lies in the peculiar material, the special brush and ink effect, the unique painting pattern, the poetry, calligraphy, painting and seal cutting, the mounting style, the composition and the technique and style of the painter. Only those, who have been constantly working on the paintings, and making contact with Chinese Xuan paper and ink for long, can write the articles about the formal beauty of Chinese paintings, express the true experience and profound artistic connotation in the practice of painting creation. This has guiding 
significance in the creation of paintings. Chinese ink painting, boasting a history of more than a thousand years, represents the wisdom and talents of Chinese people. Moreover, it has been the crystallization of traditional Chinese culture and art since a long period of time. Nowadays, Chinese ink paintings are increasingly cherished by Chinese and foreign people.

\section{Four Treasures in Chinese Paintings}

The four treasures are the material basis of Chinese paintings, and its physical form determines the development direction of Chinese paintings. Ancient Chinese paintings appeared in the places where sacrifices were gathered for the first time, and were usually made on the cliffs of caves or the walls of temples with the original paint. At the beginning of the agricultural civilization, the painting was gradually drawn on the animal skin, fabric and silk.

\section{(I) Chinese Xuan Paper}

The most primitive ancient paper was produced in the Western Han Dynasty. In the Eastern Han Dynasty, the papermaking technology became matured gradually. Various ancient papers experienced the period from the initial use to the extensive use in the Wei, Jin, Southern and Northern Dynasties and the Tang Dynasty. Chinese Xuan paper, as the most famous one among Chinese handmade papers, is called "Four Treasures" together with Huzhou writing brush, Huizhou ink and Duanxi inkstone. Chinese Xuan paper is a bast paper, with sandalwood bark as an important raw material. The word "Chinese Xuan paper" first appeared in the Notes of Past Famous Paintings by Zhang Yanyuan of the Tang Dynasty, "Chinese Xuan papers are processed with wax by the paper owner for painting in future." In the Five Dynasties and Ten Kingdoms Period, Xuanzhou region produced the famous "Chengxin Hall paper", which was described as that "it is pure white, hard, thin and smooth, thus making a great flutter." Xuan paper enjoys the reputation of "Paper with A Thousand Years' Life" and "King of Papers". It is the carrier of the inheritance of Chinese calligraphy and painting art, and is also a beautiful treasure in the history of Chinese culture. The celebrity's calligraphy and painting, historical documents, etc., which were written, printed and imitated on Chinese Xuan paper since the Ming dynasty, are as intact as before, and passed down to the present, as long as they have been properly preserved.

From the analysis of historical famous painting papers, in the Tang, Five Dynasties and Northern Song Dynasties, most painters continued to use the boiled and scoured raw silk and the processed bast paper to paint. Until the middle and late Northern Song Dynasty, Mi Zhizhang painted the rain landscape, and set a precedent 
of painting with plain white raw silk. Later, the painters painted both with the processed and unprocessed papers. Xuan paper is mostly unprocessed paper, used for freehand and semi-freehand paintings, and a small amount of processed Xuan paper is used for elaborate style paintings. It is said that Chinese Xuan paper was founded in the early Tang Dynasty, and was listed as a tribute by the Tang Dynasty. In the Yuan Dynasty, Chinese Xuan paper was widely used. Modern Chinese Xuan paper originated from the Yuan and Ming Dynasties, with sandalwood bark and sandfield straw as the main raw materials, and has continued up to now (CAO Tiansheng, SU Cheng'ai, 2016). The Southern Song Dynasty and the Yuan Dynasty saw the emergence of a large number of paper-based Chinese paintings. Compared with the painting history of the Northern and Southern Song Dynasty, the Northern Song Dynasty's paintings are mostly silk scrolls. In the Southern Song Dynasty, paper paintings accounted for a certain proportion. In the Yuan Dynasty, a majority of paintings were drawn on papers. From the Ming and Qing Dynasties to this day, paper painting has dominated Chinese paintings. This paper is very different from the paper used in the western painting. It has the advantages and characteristics of water and ink absorption, ink dispersion and brush fixation, strong coloring power, thin and soft texture, etc., which is Chinese Xuan paper in the "Four Treasures" often mentioned by later generations. It was first produced in Jiangxi, Sichuan, Eastern Zhejiang, Southern Anhui and other places. In especial, the paper produced by Xuancheng, Anhui is the most exquisite. The making material and process of Xuan paper are very particular.

Take the shrimps painted by Qi Baishi, the camels painted by Wu Zuoren, and the horses painted by Xu Beihong for example. If they are not painted on good Chinese Xuan paper, it will not have the effect of dripping ink, moist and transparent way of painting and clear penetration. Looking at the landscape by Huang Binhong, if it is not painted on good authentic Chinese Xuan paper, it is impossible to have layers of coloring, real in virtual, white in black, and moist effects. If the landscape by $\mathrm{Fu}$ Baoshi is not painted on the bast paper similar to Chinese Xuan paper, it cannot produce a kind of vigorous and ancient, sturdy and simple effect, and has the effect of diffuse permeation of the bast paper grain. If the "Landscape Painting of Ice and Snow" in the northeast is not painted on the authentic Chinese Xuan paper, it can't produce the ice and snow miracle effects of light in light, clear layers, transparent and superposed stroke mark. These effects are attributed to the paper. It is a kind of form conversion, and a special ink image, which brings an inexpressible beauty, an object produced beauty, and a high-level visual beauty. This beauty is the formal beauty. 


\section{(II) Ink Brush}

The ink brush, as a unique painting tool in Chinese paintings, is divided into three categories: soft, hard and mixed hairs. The hair is often made from the hair of wolves, sheep, badgers, rats, rabbits and other animals. The use of ink brush has a long history. According to textual research, there are traces of the use of ink brush in the scribed lines of the Yin Ruins' Oracle Bone Inscriptions of the Shang Dynasty. The creation and use of ink brush begin with writing, which is also the source of painting. While the ink brush is wielded on Chinese Xuan paper, different performances of ink brush can produce different lines or ink effects. In general, the hard hair can produce vigorous and forceful, energetic and heavy, dry and flying effects. The line drawn by the soft brush has firmness in gentleness, ink in the line, line in the ink and water-ink blending. The hard brush is easy for writing, and the soft brush is easy for coloring.

(III) Ink

Ink is a thick, smooth, moist and exquisite black liquid used by the ink brush on the Chinese Xuan paper, and is ground with the ink block in the inkstone. The brush dipped in ink and water can bring out different shades of ink, such as dry, thick, heavy, light and very light, to draw and create on Chinese Xuan paper. Different inks can produce different ink properties, the amount of water and the harmony of ink and color can produce different visual effects. The old and new inks also have different effects. The overnight ink that has not deteriorated is soft, gray and moist. Since the last decade or so, Wu Shanming of Zhejiang has been painting figures and landscapes with overnight inks, forming his own style, which has influenced Jiangsu and Zhejiang.

\section{(IV) Inkstone}

An inkstone is a tool for grinding ink and a platform for ink production. The brush has the brush property, ink has the ink property, so do the paper and inkstone. The painter's satisfaction with the ink ground has a great relationship with the quality of the inkstone. Ancient literati attached great importance to the inkstone with high quality. Without the improvement and promotion for brushes, ink, papers and inkstones by Chinese painters and craftsmen of "Four Treasures" from generation to generation, it is impossible to have the present appearance of Chinese paintings, as well as the current aesthetic inheritance with "writing" as the core and ink painting as the noumenon; it is impossible for Chinese painting to have the current unique art form and to be extensively favored by Chinese. Therefore, to study the artistic beauty of Chinese paintings, we must first familiarize ourselves with the painting materials applied to Chinese paintings, and the quality attributes and characteristics of different 
materials. Otherwise, we will lose the artistic source to study and discuss Chinese painting art.

\section{Chinese Xuan Paper}

\section{(1) Raw Material}

Papermaking is one of the four great inventions in ancient China. China has a long history of papermaking, which can be dated back to more than 1,000 years ago. Generally, paper refers to the sheet with uniform thickness made from specially treated plant fibers (except that chemical fibers are used to produce the special paper) after a series of processes (Zhang Jianzhou \& Liang Luning, 2018). Therefore, the most important raw material in paper is plant fiber. The plant fiber is divided into wood fiber (needlebush, hardwood), grass fiber, bast fiber and cotton fiber. The use of grass raw materials and bast raw materials for papermaking is a major feature of papermaking in China. At the same time, China also has the longest and most successful history of using grass and bast plants as raw materials for papermaking. Since the use of bast fibers for papermaking more than 1800 years ago, the bast fiber has been mainly used for calligraphy and painting paper and Chinese Xuan paper so far. In addition, until the end of the 20th century, grass raw materials still accounted for more than $60 \%$ of China's papermaking raw materials. The identification of the formation time of calligraphy, paintings and documents has always been the key and difficult point of forensic science, especially the identification of "paper age".

Since Cai Lun's papermaking in the Eastern Han Dynasty, the mulberry bast, cannabis sativa and ramie have been the main pulp species for the production of calligraphy and painting paper and Chinese Xuan paper. They are still used in the field of calligraphy and painting paper and Chinese Xuan paper. The main raw material of the early Tengchong calligraphy and painting paper was the mulberry bast. Tengchong calligraphy and painting paper is also known as Tengchong Xuan paper (referred to as "Teng Xuan"). It is a handmade paper produced in Tengchong County, Yunnan Province. Later, straw and bamboo were added, and the traditional raw materials are still used today. Tengchong calligraphy and painting paper is developed by Tengchong Xuan Paper Factory in 1973 on the basis of the original bast paper, using the local edgeworthia gardneri bast and straw as materials and consulting the production experience of Jing County Xuan Paper of Anhui Province. Its output is second only to Anhui Xuan paper in national calligraphy and painting paper, and it is sold well at home and abroad.

According to Gezhi Jingyuan, as early as 1600 years ago in the Jin Dynasty, Zhang Mao of the Eastern Jin Dynasty began to apply bamboo to papermaking, 
which made China the first country in the world to use bamboo paper. In the Song Dynasty, bamboo began to be used to produce paper, but due to the use of nonboiled materials, it developed slowly in output and quality. Since the use of boiled materials in the Yuan Dynasty, it developed rapidly, and a large number of painting and calligraphy papers begun to use bamboo pulp paper. Especially in the late Ming and early Qing dynasties, it reached the heyday of bamboo paper. Daqian calligraphy and painting paper, formerly known as Sichuan Lianshi Paper, also known as "Jia Xuan", Jiajiang Paper, Jiajiang Xuan or Jiajiang Chinese Painting Paper, is referred to as Daqian Paper. It is produced in Jiajiang County of the Qingyi River basin at the foot of Mount Emei in the western part of Sichuan Province, and has a history of several hundred years. As early as in the Ming and Qing Dynasties, the handmade paper produced in this area was listed as "tribute paper" and was designated as "paper for imperial examination". Fujian Liancheng Paper also used bamboo as raw material, was originally produced in Shaowu of Fujian, and then spread from Fujian to Jiangxi, Sichuan, Zhejiang, Yunnan and other places. It began to be used in the Qing Dynasty as the court paper for writing and printing, and became famous at home and abroad. General Catalogue of Imperial Collection of Four Divisions of the Qing Dynasty was printed on Lianshi paper. In addition, Fuyang Yuan Paper, which is one of China's handmade papers, is mainly made from phyllostachys pubescen and phyllostachys nuda. The paper is thin and delicate, and presents light yolk yellow; Fuyang Jingfang Paper, also known as Jingbang Paper, is mainly made from tender bamboo, processed by boiling method, and the pulp is naturally bleached and made of bamboo curtain. The paper is white, uniform and smooth. After the liberation, with the reform of production equipment, it is particularly suitable for the development of bamboo pulp production. The output of bamboo pulp has grown from a few tons at the beginning of liberation to 1.94 million tons in 2010. Bamboo pulp fiber becomes an important raw material for pulping and papermaking in China.

Mulberry is an economic crop developed earlier in China. After used in papermaking, mulberry bark has quickly become the main raw material for papermaking, and is still one of the main raw materials for Chinese calligraphy and painting paper. It is reported that there are about 12 species of mulberries in the world, and there are mainly 5 species in China. Mulberry bark is the endothelium stripped from the tender stalk or branch bast zone of mulberry. The mulberry is the oldest and most economical horticultural plant in China. The leaves can be used for silkworm feeding, the stalks can be used as medicine, the mulberries can be used for wine or food, and the barks can be used as substitute for hemp, and as excellent raw 
material for papermaking. It is distributed in Hebei, Shandong, Shaanxi, Sichuan, Jiangxi and Zhejiang. Qian'an Gaoli Paper is a handmade paper produced in Qian'an, Hebei. There are two kinds of pulp ratio for making Gaoli paper, one is $100 \%$ mulberry bark, and the other is $40 \%$ mulberry bark and $40 \%$ deckle edge. Its production cycle is about 10 days from barks to finished products. The appearance of Qiang'an Gaoli Paper is characterized by the obvious netting, especially the prominent thicker white cotton line watermark. On the basis of producing this paper, Qian'an developed the "Qian'an calligraphy and painting paper" from the production experience of Jing County Xuan Paper of Anhui Province, which won the praise of calligraphers and painters, and the praise of "Southern Xuan Paper and Northern Qian'an Paper".

Sandalwood bark became famous all over the world during the Xuande period of the Ming Dynasty, because the quality of pteroceltis tatarinowii bark paper in Jing County, Anhui Province surpassed Jiangsu and Zhejiang papers. Pterocarpus indicus, dalbergia hupeana and symplocos paniculata (also called pteroceltis tatarinowii) are commonly found in China. Only pteroceltis tatarinowii with white wood and caesious bark is suitable for papermaking. The bark is rich in fiber and easy to bleach, so it is an excellent raw material for papermaking. The Chinese Xuan paper made from pteroceltis tatarinowii has strong adsorption and long life, is not easy to deform, anti-aging, anti-insect, and the paper has six characteristics of thin, light, soft, tough, fine and white. Xuan paper of the Song and Yuan dynasties (Cao Tiansheng, 2017) is mainly made from pteroceltis tatarinowii. Such paper can help the calligrapher and painter to achieve the special artistic effect of changeable shade and strong ink absorption during the painting and calligraphy creation. It is the raw material of China's famous Xuan paper. According to the fiber ratio of sandalwood bark and straw, Jing County Xuan Paper can be divided into three categories: straw paper, bark paper, and special bark paper. Jing County Sandalwood Bark Xuan Paper still ranks first in China's Xuan paper. the "sandalwood bark" fiber, since its appearance in the Ming Dynasty, has been unabated and is still popular today.

The earliest record about the use of straw for papermaking appeared in the Northern and Southern Dynasties, but they were all straw papers, which cannot be handed down. Straw was not blended in the raw materials in the early stage of Chinese Xuan paper production. Later, in the processing of bark materials, the Xuan paper maker found that the sandfield straw locally produced is of fine quality. The fiber form is quite similar to the pteroceltis tatarinowii bark, but the toughness is slightly inferior, and it plays a role in enhancing the softness and flexibility in Chinese Xuan paper. The Chinese Xuan paper is made by mixing the pteroceltis tatarinowii 
bark fiber with the straw fiber. The bark is the backbone, and the straw is the muscle of Chinese Xuan paper. That with more bark is tough and is called bark Xuan paper, and that with more straws is soft and is called straw Xuan paper. The straw fiber was most popular in the production of Chinese Xuan paper in the Ming Dynasty, and the mixed use of bark and straw became gradually matured in the Ming Dynasty. The most representative was the Xuan paper produced in South Anhui region at that time. Such Xuan paper was made from the mixed pulp of local sandfield straw and pteroceltis tatarinowii bark. Xuan paper of the Ming Dynasty can keep intact for a thousand years and has been passed down to this day. According to historical records and experts' tests on ancient Chinese Xuan paper, the production of Chinese Xuan paper with straw and pteroceltis tatarinowii bark mixture began in the Ming Dynasty and became popular in the Qing Dynasty.

(II) Artistic Status

Scholars and poets of the past dynasties praised Xuan paper, but rarely mentioned the processing technology and process of Chinese Xuan paper. The processing technology and production process of Chinese Xuan paper are mainly inherited by that the master accepts apprentices. There is no large-scale and systematic production. This method has continued to this day. "The production technology of Chinese Xuan paper" was included in the first intangible cultural heritage list in China in October 2006 (Lu Qing, 2018), and then listed in the representative list of human intangible culture by UNESCO in October 2009. In December 2010, "Research on the Rescuing Excavation and Sorting of Traditional Production Techniques of Chinese Xuan Paper" was listed as a major bidding project by the National Office for Philosophy and Social Science of China. These examples fully illustrate the cultural status of Xuan Paper in China and even in the world. The opening ceremony of the 2008 Olympic Games is still fresh in our memory. A beautiful picture on the opening ceremony fully demonstrates multiple links like paper-spreading, ink grinding and painting. At the same time, in order to reveal the traditional culture of China in a better way, Director Zhang Yimou specially added the close-up view of papermaking by hand, which reflects the great significance of the traditional manual papermaking technique in the process of inheriting the entire Chinese civilization and in the whole world.

\section{(III) Production Technology}

China's Xuan paper is well-known at home and abroad, which is determined by its excellent quality (Liu Renqing, 2008). The fine traditional Chinese Xuan paper process goes through 108 complex processes. Firstly, the straw and the pteroceltis tatarinowii bark are treated separately by airing, and then the prepared pulp and straw 
pulp are mixed for papermaking (Wu Shixin, 2013). Before papermaking, a kind of plant vine juice (Actinidia chinensis Planch juice) will be added to the mixed pulp in the ancient method of making Chinese Xuan paper. This juice mainly plays the role of floating pulp and homogenate, and is also important in the manual paper separation.

Chinese Xuan paper is divided into the unprocessed, processed and halfprocessed. Different papers are different in size, thickness and quality, and have different effects on painting. In the creation of Chinese paintings, Li Keran talks about "paper first", because different papers have different ink and form effects due to different origins, different materials, different thicknesses, different absorption capacities, different paper grains and textures. This difference can, according to different objects, produce different formal beauty through the content.

(IV) Production Rules

Chinese Xuan paper is known to be made from China's specialty sandalwood bark and sandfield straw. It is made by traditional microbial decomposition and mild digesting pulping method and natural oxygen bleaching. From the principle of papermaking, the following rules should be mastered.

First, in the selection of raw materials, the characteristics of the fiber raw materials is mastered, while increasing the surface area of the ink absorption of the fibers, to express the different shades of ink color and layers, thus retaining the brush marks and ink marks, and withstanding strict requirements on superposition, ink breaking, and so on. Second, it is necessary to prevent the curing and hydrolysis of cellulose, without changing its chemical structure and properties, and prevent the paper from hardening, which may affect the water and ink absorption, and cause the painting to turn yellow in future. Third, the hemicellulose content is reduced; microbial decomposition is the most effective method for straw pulp, the ratio of cellulose content to pentose can be more than 5 times, and the paper is soft, tough, and has strong ink rhyme. Fourth, the thermolabile sizing dispersant is selected to keep the paper in good appearance. It retains the characteristics of micro light sizing, so that the Chinese Xuan paper will not, like the blotting paper, diffuse and penetrate excessively. Fifth, for "A Thousand Years' Life", we should summarize the experience of making Chinese Xuan paper, pay attention to the material of pulping and papermaking equipment and water quality, to prevent residual harmful ions, and use natural synthetic carbonate as protective agent to improve paper life.

(V) Paper Properties

The color of traditional Chinese Xuan paper is white and pleasing. With over $90 \%$ whiteness, it is not easy to yellow and discolor for a long time, and can maintain 
the true color of painting and calligraphy for a long time. Chinese Xuan paper is soft and tough, and can be restored without folding mark after folded. When painted by the Chinese painter, the paper is not broken. After soaked with ink, it will not bulge after drying, and the tip of the brush does not rub off the microfiber. Chinese Xuan paper has a variety of watermark patterns. Chinese Xuan paper has different matching ratios. That with more bark is called bark Xuan paper, which has silky cloud flowers in perspective. Unprocessed Xuan paper with more straw has a good uniformity, and is the main material used for general painting and calligraphy, mounting painting and calligraphy. Other Xuan paper, with different thickness, includes single layer, double-layer, and also has different sizes. Xuan paper also covers a variety of processed Xuan paper, and that with color and pattern after coating processing.

Chinese Xuan paper also has the four characteristics of durability, ink embellishment, texture stability and insect resistance.

Durability. Durability is demonstrated by the ability to withstand the test of time and good resistance to damage. Experts Liu Renqing and Qu Yaoliang, respectively in 1983 and 1985, selected the special bark single-ply Xuan paper, fourfoot bark single-ply Xuan paper, four-foot straw single-ply Xuan paper produced by Jing County Xuan Paper Factory in 1983, and $50 \mathrm{~g} / \mathrm{m}^{2}$ coated art base paper, $60 \mathrm{~g} / \mathrm{m}^{2}$ (lightweight) coated paper, $70 \mathrm{~g} / \mathrm{m}^{2} \# 1$ writing paper and $48 \mathrm{~g} / \mathrm{m}^{2}$ newspaper respectively produced by Tianjin Papermaking No. 7 Factory, Beijing Papermaking No. 1 Factory and Jilin Paper Mill in the fourth quarter of 1982; these papers were treated by constant temperature and humidity, and the aging test was carried out after determining the main physical indicators as benchmark; it was found that compared with the coated paper, writing paper and newspaper, "Chinese Xuan paper has the best durability, and its life (simulated artificial aging time) can reach 1050 years or more" (GU Yunfang, 2018). They believe that "the good durability of Chinese Xuan paper has a close relationship with the $\mathrm{pH}$ value of the paper." "Another reason is that it is inseparable from the excellent properties of the pteroceltis tatarinowii bark fiber, which is durable and not easily damaged." Painting of Plum Blossom by painter Tang Bohu of the Ming dynasty preserved in the Palace Museum, was painted on Xuan paper about 500 years ago. Sun Xinsheng, from Qingzhou Museum of Shandong Province, used Chinese Xuan paper for the mounting of his Palace Examination Paper of No. 1 Scholar Zhao Bingzhong in the Period of Emperor Wanli in the Ming Dynasty, which has a history of 400 years. The Imperial Chunhua Pavilion Carving Xuan Paper of the Early Qing Dynasty in The Palace Museum 
provided by Zhang Shufen also has a history of more than 300 years. Apparently, the statement of Xuan paper with "A Thousand Years' Life" is not exaggerated.

Ink embellishment. Compared with other kinds of calligraphy and painting papers, Chinese Xuan paper has the best ink embellishment, which is also the biggest feature of Chinese Xuan paper. The ink embellishment of paper refers to that when creating paintings and calligraphy works with water and ink, the diffuse edge of the ink mark should be symmetrical, the inking should have strong penetration and adsorption, and the ink color should have clear shading, clear layer and rich stereoscopic impression. In various characteristics of Chinese Xuan paper, ink embellishment, as the most prominent one, is one of the most important quality indicators required by Chinese calligraphy and painting for Chinese Xuan paper. According to the study of Liu Renqing and Qu Yaoliang on the adsorption properties, fiber morphology and internal components of Chinese Xuan paper, the pteroceltis tatarinowii bast fiber has good uniformity, thin wall and moderate softness, especially after natural drying, on the bast fiber cell wall are many wrinkles parallel to the long axis of the fiber, which is an important condition for good ink embellishment; the ink embellishment of Chinese Xuan paper depends on the accumulation of calcium carbonate $\left(\mathrm{CaCO}_{3}\right)$ between the wrinkles on the cell wall of the pteroceltis tatarinowii bast fiber; the ink embellishment effect of Chinese Xuan paper is mainly manifested in the vertical and horizontal differences of the diffusion after the ink absorption on the paper surface, the depth of the ink absorption and the gradation of the clear shading. Special bark Xuan paper has the best ink embellishment, mainly due to containing more raw materials of pteroceltis tatarinowii bark. Therefore, to some extent, the more sandalwood bark in Chinese Xuan paper, the better its ink embellishment.

Texture stability. Compared with other kinds of calligraphy and painting papers, Chinese Xuan paper is most resistant to deformation. After the paper surface of Chinese Xuan paper is subjected to ink, its deformability is extremely stable, and the phenomena of fluffing, arching and curling are almost negligible. This is mainly because the pteroceltis tatarinowii bark fiber has a high regularity, and there are many voids between the fibers, so the bonding force between the fibers is small, and the paper is not easily deformed. Second, the content of hemicellulose in Chinese Xuan paper is small, and hemicellulose is the most easily wet-swelling part of the fiber. The smaller the content of hemicellulose, the smaller the deformation. Third, the plant sizing and fillers used in the production act as separation between the fibers, reducing the change in fiber binding force when the Chinese Xuan paper shrinks after inking. 
In addition, the longer the storage time of Chinese Xuan paper, the less likely it will deform.

Insect resistance. Chinese Xuan paper is also very resistant to insects. However, if Chinese Xuan paper, its calligraphy and painting products, and books with Chinese Xuan paper as carrier cannot be properly kept, they will be also attacked by insects. Therefore, in addition to the use of Chinese Xuan paper, we must think of ways to protect Xuan paper carefully, to ensure the long life, long-term preservation and integrity of Chinese Xuan paper.

\section{(VI) Subjective Criteria for Identifying Papers}

There are hundreds of varieties of Xuan papers and processed papers, each of which has its specific use. For specific consumer objects, if making elaborate style calligraphy and paintings, you should use the Xuan paper that has been processed with alum, also known as "alum Xuan paper", but cannot use the "unprocessed Xuan paper"; if drawing Chinese paintings and writing calligraphy, you should use the unprocessed Xuan paper; if drawing large-scale paintings and writing large characters, you should use $4 \mathrm{~m}, 5.12 \mathrm{~m}, 6 \mathrm{~m}$ or even larger Xuan paper according to your needs; For example, beginners use the bark Xuan paper at the beginning, which not only causes waste, but also makes it difficult to wield the brush. It is better to use the imitation Xuan paper first, and then use the bark Xuan paper after mastering the brush technique. Only by making clear the intention of using Xuan paper can you choose the desired Xuan paper, and the Xuan paper that suits your intentions is a good Xuan paper. As for the subjective criteria of Xuan paper and the adaptability of using Xuan paper, calligraphers or painters, from the original refusal to use Xuan paper to the use of Xuan paper, cannot adapt to the ink embellishment nature of Xuan paper at first, and grasp the degree of inking, etc., because they cannot grasp the characteristics of Xuan paper. Only by knowing clearly the characteristics of Xuan paper in the long-term use, can we use freely and control truly Xuan paper to create the best paintings and calligraphy. It should also be pointed out that the quality of the Xuan paper produced and sold by various Xuan paper manufacturers is still different due to various reasons. These differences do not result in obvious quality advantages and disadvantages, and some can be ignored, some can only be distinguished by experts, and some can only be realized in the actual use.

"Yesterday the person of court came over, and sent two-axes Chengxin Hall papers, I opened the papers, seeing that paper is as smooth as spring ice, as dense as cocoon, and I felt surprised extremely and lingered." This was indited by poet Mei Yaochen of the Song Dynasty, who felt quite pleased when receiving two Chengxin Hall papers supervised by the Southern Tang emperor Li Yu from Ouyang Xiu (Sun 
Chunli, 2018). From this, we can see the status of Xuan paper in the minds of ancient scholars. Therefore, the Xuan paper with the reputation of "Paper with A Thousand Years' Life" is not only a kind of art circulation and inheritance, but also the source of China's unique cultural charm.

\section{Art on Chinese Xuan Paper - Chinese Ink Painting \\ (I) Ink Color}

The concept of Chinese painting appears after the western painting enters China. Chinese paintings before the Tang Dynasty were not unicolor, and the imperial-court decorative paintings of the Song Dynasty focused on colors and lines. There is a mindset that people think it is the western painting when seeing colors, which is actually wrong. Color is not unique to the western painting; it also belongs to the Orient. Color is the basic factor of any painting.

The ancient painting theory "Application of Colors according to Categories" is for the intrinsic color, meaning that the painter will paint the mountains, rocks and trees according to their own color. In the literati painting, the picture is basically unicolor, and the shading of the ink color distinguished with water acts as the color. Here, the "Application of Colors according to Categories" is to paint a similar light color on the basis of the ink color. There are so many colors in Chinese painting, why to use one ink color to dilute the shade to serve as color? In the distant Neolithic Age, there were patterns drawn in black on the preserved colored pottery or black pottery, which indicates that black was one of the earliest colors used by Chinese ancestors. On the colored pottery jar with stork, fish and stone axe pattern that was unearthed from the Banpo Remain 7000-9000 years ago, the large circle pattern, curly pattern, hook pattern and frog pattern on the colored pottery of the Majiayao culture all reflect the art of patterns drawn in black. This shows that the clan had a conscious concept of worship for black. Therefore, in the long history, the aesthetic concept of black worship has affected many aspects of Chinese people's social life, especially in the field of painting. Black is the primitive instinct perception of ancient humans to natural colors, and this perception of the ancient Chinese is particularly strong and sensitive. The black worship, and the fact that black is incisively and vividly exerted in ancient paintings, have contributed to China's unique style and genre of ink paintings. This is closely related to the unique aesthetics of black worship and national aesthetic taste of Chinese ancient people. In addition to cultural and psychological factors, China is an official-based country, and the interest of the scholar-officials promotes the development. 
Chinese painting has experienced two forms in the process of development: ink painting and green landscape painting (Yin Xing, 2017). Due to the simplicity and naturality of ink painting's ink color, the ink paintings with ink color as the expression method are more admired by the public. Ancient literati often expressed the elegant, natural and innocent artistic tastes through ink paintings. In Chinese ink paintings, ink color is the only and extremely important visual element.

In traditional Chinese paintings, color is auxiliary and is a supplement to the inaccessible color. An ancient painting theory goes that "the ink is divided into five colors" (Yin Xing, 2017), which means that after the ink color is divided into shades, it is as rich and beautiful as the colorful colors. In ancient times, a painter once described the expression technique of ink paintings, "The vegetation is luxuriant without green color; the cloud and snow are white without white color; the mountain is verdant without green color; the wind is dynamic without five colors; five colors are available with good ink, which means perceiving the artistic conception." (Sun Chunli, 2018) Ink has five colors, that is, five ink techniques of dry, thick, light, withered and wet, which may reflect the changes of grass, wood, mountains, rocks, tiles and snow without any other colors. If no good Xuan paper is available, even with the good ink, it is difficult to give play to the artist's artistic expertise. Because the poor paper will make the dry, thick, light, withered, wet ink color dissolved into a flat piece, become a dull brush mark, or a combination of thick and light lines. The ink color includes "penetrating ink" and "floating ink". If the artist's original work is directly painted on the uncoated Xuan paper, the ink and color will permeate into the texture of the paper, which is called "penetrating ink". High-simulation calligraphy and paintings must be treated with surface sizing in order to scan without any difference, so that the ink is floating, that is, "floating ink". The breath that floats on the surface is more obvious on the edge of the calligraphy and paintings.

Wang Shu of the Qing Dynasty said that "Using water in the inkstone to grind ink will make the brush unsmooth and the ink stagnant, and fresh water is the best. The wide inkstone with fine surface washed every night can make water and ink uniform. It is wonderful to take fine and glossy water with Duanxi inkstone and grind ink with She inkstone. It is necessary to use the soft brush when writing on the hard paper and the hard brush on the soft paper." (Yang Ermin, 2018) Zhao Huanguang talked about the relationship of brushes, ink, paper and inkstone as follows, "The soft brush is suitable for the hard paper", "For soft hair, thick ink is slightly used to produce the best work, and the slight ink is thickly used to produce the poor work", "For ink on the hard paper, the ink should be surplus; the brush with thick ink should not be dried", "The ink should be like paint, the paper like bark, the inkstone like jar, 
and the palm like wooden chick; if anyone is not eligible, it will produce the vulgar works" (Chen Zhiwei, Xie Chongkai, 1986). Therefore, good papers and good inks are interdependent and indispensable. We have experienced that if the painter finds the good brush and the good paper in painting, he has great interest in painting and fondles admiringly. However, if the painter paints with brush on a poor paper, he will lose the interest in painting completely; or he may feel not bad at first in painting, but the ink becomes gray, the shading is blurry, and the gradation is gone after the ink is dry.

\section{(II) Artistic Conception of Brush and Ink}

The technique of brush and ink refers to the brushwork and the ink technique, which are the means of Chinese paintings. In any Chinese painting, the object is represented by brushwork and ink technique. For Chinese painters, "brush and ink" are the effort, the artistic accomplishment, and the artistic attainment that the painter pursues and reflects constantly, and represents the artistic level of Chinese painters.

In the creation of Chinese paintings, it stresses "likeness and unlikeness" in expressing and reflecting any object. Unlikeness means deceiving the world; for likeness, it is tasteless and not vivid without brush and ink. For Chinese paintings, especially professional paintings, its goodness and grade depend on the use of brush and ink. Good and beautiful brush and ink can touch people and further sublimate into a formal beauty. The brush is linear, the ink is planar, and the short and small brush and ink are points. Points, lines, and planes are painting forms, and the basic elements of visual image combination. In creating on Xuan paper, especially on the unprocessed Xuan paper, the painters have experienced that the brush tip, the brush belly, water content at the brush root, the ink amount, the way of writing, the central or the side brush technique, the strength of wielding brush, lifting and pressing, the speed, smoothness, cadence, transition, agreeable movement, inverse movement, gathering movement, and diffuse movement can produce different artistic effects. Like the human body, the brush should have tendon, flesh, bones and vitality. Lines should be flat, round, stable, thick and changeable. Flat, as if drawn on sand with an awl; round, like "brasenia strip", "water stains on the wall"; thick, like "dropstone on the mountain"; change, square in flat, round in square, false or true complement. All these are transformed into an image that sublimates into a visual beauty. The ink technique is the same. Huang Binhong said that there are seven ink techniques, namely, thick, light, breaking, splashing, accumulated, dry and overnight. Different brush marks, traces, and penetration of different ink techniques on different Xuan papers will present different visual images and produce different beauty. In the practice of water and ink, the water amount, the ink thickness, breaking ink with 
water, breaking color with ink, breaking ink with color, flushing, accumulating, dissolving, splashing and other means, the length of time the brush stays on the paper, the speed of the brush movement can produce unpredictable and indescribable artistic effects. This is the beauty of formal charm, and the formal beauty of Chinese paintings.

The expression form of Chinese painting is "brush and ink". The connotation that "brush and ink" express is real, because the performance of the painter's ink and brush is visible in the works: shading, dry, line-drawing, cun (wrinkle), dotting, dyeing, splash-ink, accumulated ink, breaking ink, dry ink and other painting methods with brush and ink. At the same time, "brush and ink" are also virtual, because it is not only a visual technique of expression, but also a cultural feeling. When appreciating the painting with this cultural feeling, you can understand the ink color taste and artistic conception presented by various techniques of the painting. Mr. Wan Qingli believes that "Brush and ink are not only abstract points, lines and planes, or the means of modeling attached to objects. They are the traces of a painter's heart, the appearance of his character, the revelation of his temperament, the display of his aesthetics and the mark of his knowledge."

Chinese paintings that follow the ancient aesthetics of black worship of ancient China mainly represent the painted objects with ink color and the "blank left" of the picture. White, as a contrasting color of black, can set off and extend the artistic conception of the picture of the ink color. This coincides with "setting off the black with blank" by Laozi and Zhuangzi, and also agrees with the picture effect of "setting off real with virtual", so that the picture presents an infinite imagination and interesting taste. “Xuan” (Chinese character “玄”) representing black reveals the image that a seed is covered with soil and will break the soil and sprout. The meaning of "Xuan" actually represents a bud. Laozi believes that "virtual" and "real" are the "origin" and "mother" of all things, and the combined action of "virtual" and "real" produces all things on earth. Therefore, the "virtual" and "real" presented in Chinese paintings reflect the artistic conception access to "Tao". Therefore, the "brush and ink" presented in Chinese paintings is the combination of the virtual and the real, and the dual combination of the implicit artistic conception and the explicit ink color, thus, it has a very high aesthetic value.

\section{(III) Composition}

Composition is the biggest form of Chinese painting's picture. The stronger the sense of form, the stronger the appeal of the picture. Chinese painting has the most abundant internal and external form of composition. The ancients used five Chinese 
characters of “之, 由, 甲, 则, 须” to basically solve the inherent composition requirements of Chinese paintings, like the layout, primary and secondary, contrast, balance, density, opening and closing (Mao Wanbao \& Huang Jun, 2009). In the external form, Chinese paintings are divided into such styles as central scroll, vertically-hung scroll, banner, long roll, doufang (square paper for painting and calligraphy), panorama, hand scroll, fan and album according to the mounting and using function. The composition form of five-character composition method and the mounting form of Chinese painting produce the look-up, overlooking and profound beauty. For example, the central scroll “甲, 由” shaped compositions are sublime and integral, and have the towering and admiring beauty. The deep and profound “之” shaped composition is varied, rich in layers, and circuitous, and has the beauty of high and distant mystery. The composition of the long scroll of the banner is long and flat, with the mountains and water stretching far and wide; the rich scenery stretches the heart and contributes to the leisurely interest. The circular fan composition is thick and changeable. The panorama composition, independent respectively but complete on the whole, is magnificent. Different forms of composition produce different visual impacts and visual effects, which is the beauty of form generated by the unique composition of Chinese painting.

(IV) Technique Pattern

In terms of technique pattern, the pattern is a model, a procedure and a established standard, to form certain format or routine. The technique pattern of Chinese painting is to refine and summarize the objects of nature in accordance with the laws of drawing style and gesture, to form a certain format and routine. For example, bamboo leaves can be painted with two-leaf, three-leaf, four-leaf, five-leaf and multi-leaf. Chinese Hemp Agrimony is painted by the three alternating strokes. In the landscape paintings, the painting of mountains includes line cun, plane cun and point cun. Line cun is often used in the southern landscape, such as Pima Cun (the technique of wrinkling). Plane cun is frequently used in the northern landscape, such as Fupi Cun (axe-cut stroke). The technique books such as Mustard Seed Garden Paining Biography in the Qing Dynasty are not only the tool books for beginners to learn painting, but also good teaching materials for understanding the forms of Chinese paintings and mastering the rules of Chinese painting pattern. In traditional Chinese art, the ubiquitous pattern is a technical induction, a refinement, a simplification of complex objects, and an art law that must be mastered. In the creation of Chinese paintings, it is very important to master the pattern. For example, Qi Baishi summarizes the shrimp head into three strokes, the shrimp body into five 
strokes, the tail into three strokes in painting shrimps. While Xu Beihong paints horses, Huang Zhou paints donkeys, Wu Zuoren paints camels, they attempt to simplify and summarize the objects reasonably into several strokes or dozens of strokes. This stylization can present the vividness of the object and the accuracy of the structure, and the dexterity, fun, taste, line, plane, light, heavy, virtual, real, rhyme and interest effect of brush and ink. It is visual and imaginal, and is also the abstract beauty of the oriental art and the high-level beauty of art form.

Techniques often have patterns, and there are many brush and ink techniques for reference and learning. However, there is no fixed style for brush and ink. Learning brush and ink techniques is just a means, which requires the learner or appreciator to adapt, develop and shift from the function of depicting objects to expressing their own unique inner feelings after mastering the brush and ink expressive ability.

\section{(V) Combination of "Poetry, Calligraphy, Painting and Seal"}

The combination of "Poetry, Calligraphy, Painting and Seal", as the unique pattern of Chinese painting, is a synthesis of Chinese traditional culture cross-border integration and a product of the perfect combination of four kinds of art. Poetry refers to verses, ditties, odes and songs, and is the soul of Chinese painting. Calligraphy and seal are indispensable in Chinese paintings; calligraphy is the preface and postscript of Chinese paintings, and an important manifestation of the painting's charm and vigour. In the creation of paintings, the painter must have a solid foundation of calligraphy. Without the support of calligraphy, Chinese paintings are weak and vacuous. Seal cutting is the seal used by painters, including name seal, alias seal, optional seal and layout seal. The seal with bright red seal oil is stamped on the picture, which is a striking "point". Calligraphy, seal and Chinese painting style must be combined, coordinated and rationally arranged. Perfect combination and integration of the three kinds of arts can enrich the picture, and promote the aesthetic connotation of the picture and the artistic conception of the painting. It is a perfect embodiment of the formal beauty of a good Chinese painting.

Just like other arts, the appreciation of Chinese painting has its own characteristics. Only by correctly grasping the law of appreciation, and boldly attempting and studying these laws, can we ultimately achieve the highest level of appreciation.

\section{Chinese Xuan Paper and me}

(I) Origin 
I studied in Ukraine and Russia for decades, and followed the Ukrainian people's artist A.M. Lopukhov, Andrew Atroshenko, Alexander Bystrov, academician of Russian Academy of Arts and Sciences, and other masters to learn the oil painting art skills of top international artists. Years of art study experience has cultivated my artistic deposits. After graduation and returning to China, I followed the famous flower and bird painting master He Shuifa to learn Chinese painting. I still remember that as a beginner of ink paintings I would get addicted whenever drawing with a brush, I would draw dozens of small paintings continuously every day and draw a lot of beautiful natural scenery. Every time reading the painting every few days, I can still feel the joy and tender feelings in painting. In these years of studying Chinese paintings, I have become more passionate about China's outstanding traditional art - Chinese ink painting.

\section{(II) My Enthusiasm for Brush and Ink.}

I like traditional Chinese painting, and I also know well the technique of point, line and plane and the implication of the blank left. Chinese painting, as one of the few oriental traditional cultures, is originated in the Neolithic Age and has been evolving since then. Fewer and fewer Chinese people can concentrate on studying and understanding this art with profound Chinese cultural background. Chinese traditional paintings have various symbolic meanings. Understanding the various complex languages and discovering the deep meanings hidden in the painting is of great significance to appreciate and understand the works of masters.

It is well known that any painter in China may be a calligrapher, but not every calligrapher is a painter. In the Song Dynasty (11th century), the famous landscape painter Guo Xi wrote in his landscape painting theory The Elegance of the Bamboo and Spring the Landscape, "Appreciating the landscape with authentic taste elevates the value, and that with utilitarian idea lowers the value." The author figures out the importance of the individual style, and emphasizes that painting is a kind of narrative that shows the artist's attitude towards painting. Moreover, for the first time, the author made a request to the painter to strengthen his own cultivation. I know that my ink painting starts late, and I have practiced a lot secretly, spending time in imitating picture copybooks or celebrity's ink paintings, to master the basic skills of painting landscape, trees, flowers, birds and insects, so that the new works can be worth meticulous appreciation. Some people believe it unnecessary, and consider it great to keep natural and rootless, with which I cannot agree. This is not to blindly emphasize imitating the works of ancients, but to "imitate the ancients' trace, rather than their idea". We should not be just educated passively, but should discover, form and verify ourselves potentially in the process. Imitation allows me to understand 
that my plain brushwork, the direction of efforts, and the place of embellishment. Imitation is not only to copy the ancient brushwork, but to make clear the ink and brush procedure, the using rule of ink and brush in painting, as well as other series of things that cannot be found by sense and vision.

Lan Zhenghui, with his heroism, painted the work of Falling Sky, in which a huge black fell from the sky when Wenchuan earthquake happened, just like the ink is crying and shouting; the individual stone painted by Zhu Da spurts out the huge and magnificent momentum. There are many other examples of the beauty of ink painting. "Freehand brushwork" is a means, a method, a style, and the mainstream of Chinese painting creation. The style of freehand brushwork in Chinese painting is also various, some are vigorous, some are natural and unrestrained, some are concise and dignified, some are elegant and scholarly, some are relaxed and flexible, and some are careless; all kinds of freehand brushwork can bring different forms of beauty. In traditional Chinese paintings, the intangible is implied in the tangible, and the understanding of the intangible will be a long-term and in-depth process with the enhancement of the cognitive ability. Therefore, for the appreciator, it is necessary to understand the basic law of "brush and ink" before understanding the artistic conception of ink. This can help resonate with the painter when appreciating the works, experience the process from "imitation" to the free expression of the painter's feeling, and feel the mental experience "brush and ink techniques should be revealed from the heart and integrated with the heart" in creation. This can help appreciate and taste the artistic conception of brush and ink.

I love Chinese traditional art and love the art spirit in brush and ink more. Just as Xia Kejun (Mao Wanbao \& Huang Jun, 2009; Xia Kejun, 2017) describes the art of ink and brush - "magnificent volume ink". If the inner spirit of traditional ink painting is the Qi transformation, the mere pursuit of volume comes from the western shaping of the blocks, and is the lofty spirit of the west, then the magnificent volume ink has another spiritual temperament. It must be abstracted, become a form of power, and be blended with the natural elements to create a grand temperament in the brush and ink art. Excellent brush and ink works have the space of expressing life emotions. The color difference of black, white and gray indicates the emotions. These emotions form the direction of power, that is, the momentum, which is the extension and stretching of Qi. The whole picture is a contrast between huge blanks and ink blocks. Qi transformation can dispel lofty violence and sacrifice while maintaining the lofty.

(III) Painting Themes 
Plum blossom, orchid, bamboo and chrysanthemum, known as "four gentlemen", are traditional materials of Chinese flower and bird paintings, and also often appear in my works. Many ancient Chinese literati have qualities similar to those of plum blossom, orchid, bamboo and chrysanthemum. For example, plum blossom symbolizes lofty and unyielding character (Yuang Chifeng, 2018; Zheng Yihao, 2017), plum blossoms bloom alone in winter and snow, and this kind of character of plum blossom is quite similar to those high-minded people who are not afraid of influential officials; orchid symbolizes the secluded character, orchid has elegant and high-minded character without large leaves and fresh flowers, and the orchid's character is very compatible with that of the open-minded and narcissistic talented person; bamboo symbolizes firmness, although the bamboo is not strong enough, it is tough and straight, and this character of bamboo quite resembles that of the modest gentleman; the chrysanthemum symbolizes elegance, although chrysanthemum is beautiful and spectacular, but never flatters with tender and beautiful charm, only winning with elegance and firmness, and quietly blooming after other flowers have faded. The character of chrysanthemum coincides with that of the hermit who is independent and not timeserving. The literati express their aspirations with brush and ink, and manifest the unrequited ambition, the cynicism, the leisurely self-satisfaction and other emotions with the vivifying plum blossom, orchid, bamboo and chrysanthemum. The same is true for me to paint the "four gentlemen" to demonstrate the noble gentleman character.

(IV) Art Concept

Having studied and explored Chinese and western art for many years, I am committed to the creation concept of combining Chinese painting with oil painting. I hope my paintings can be artistically compatible and convey to the world the creative idea of the integration of traditional Chinese artistic conception and the formal beauty of the western art. Wang Wei, a poet and painter of the Tang Dynasty, said that "In the paintings, the ink painting is the best." (Method of Landscape Painting) Chinese ink painting expresses the theme and artistic conception using the flowing characteristics of ink and rich ink color layers. The reason why Chinese ink painting can become an outstanding representative of Chinese painting and enjoy the reputation of traditional Chinese painting lies in its emphasis on the simplicity, symbolism and naturalness of ink color, which is quite different from the decoration and modelling of color emphasized in western paintings. This forms a distinctive painting style with Chinese national characteristics. In the process of creation, I apply the two principles of traditional architecture and deconstruction to deconstruct the painting techniques in the traditional Chinese painting art structure, and to integrate 
the free and easy charm of the western painting in the peace and quietness of the traditional art, thus creating the Chinese traditional culture of the new era in the static and dynamic balance.

The diligent creation on the art road of exploring Chinese and Western integration has been the focus of my life in recent years. The appreciator can feel the strong vitality and the bold and unconstrained expression in the brush and ink from my works. One report has it that my work "pursues artistic conception, adopts modern composition, pays attention to brushwork, and combines modern forms of the western art with Chinese ink paintings in a better way." (Liu Jieyan \& Chang Hong, 2018) This should be the best affirmation of my artistic creation. More than ten years of learning and understanding of Chinese and western art is gathered in works, and the vigor of the brush technique and the artistic tension are reflected in the brush and ink, which enables me to gradually form my own artistic style. The works in the album I have published also reflect my exploration and persistence to my artistic concept.

Painting has its own unique language. It can resonate with the world and also has the dialect characteristic. I hope to create my own Chinese-Western fusion art style: narrating my root with the traditional art, satisfying the requirements of international standards for art with the academic standard.

\section{(V) Art Development}

In the creation of ink paintings, what to paint is not important in many cases. How to "paint", how to paint with "taste", "quality" and "style" are the things that painters think about repeatedly. The essential characteristics of Chinese painting include two main points: First, pictograph and freehand brushwork complement each other. The unity of opposites between subject and object generates the essential philosophy of Chinese painting, and thus gives birth to the rich and diverse unique expression methods of Chinese painting; Second, the core valueof enlightenment and education and the aesthetic value theory of expressing feelings have a relationship of dialectical unity (Chen Chiyu, 2016). The complementary symbiosis of these two painting values enables Chinese painting to assume social responsibility and play an ethical role, to delight the mind and create diverse aesthetic forms, thus promoting the development of artistic personality and style. In short, the beauty in form is the foundation and the core of Chinese painting. It is an inevitable development trend of Chinese art to make this mysterious form beauty unmysterious and spread it to more international friends.

However, the inheritance and development of Chinese painting now faces many problems. In terms of the school environment, Chinese painting is gradually 
fading out of the students' minds. Due to the influx of western thoughts and the neglect of the education system, many schools do not create the atmosphere of Chinese traditional culture, nor do they set up courses of Chinese painting. Chinese paintings with Chinese traditional culture characteristics gradually fade out of the students' minds, and many students have never been exposed to Chinese painting after graduation. In terms of social environment, Chinese painting has gradually faded among ordinary people. Under the impact of the western art, the western painting art has won the favor of most common people, especially the generation after 80s and 90s, with its nobleness, foreign flavour and fashion. The audience of Chinese painting has always been dominated by literati, and it can be called minority art. They focus on the charm and pursue the "rhythmic vitality", but it is difficult to explain in words and can only be sensed. Due to this reason, Chinese painting art is somewhat isolated from the masses in spreading, is not popular, and has a low popularization rate; in terms of international communication, the spread of Chinese paintings focuses on the works rather than the spirit, which lacks breadth and depth, and the form of communication is relatively simple. Westerners who lack the background of Chinese traditional culture can hardly have a correct understanding of the characteristics and value of Chinese paintings only through works (Chen Yuxing, 2016).

To my way of thinking, such situation should be improved from the inside out. First of all, a good campus atmosphere should be built, so that students can be influenced in person from childhood, so as to maximize the acceptance of Chinese painting culture edification. In terms of social communication, we should focus on integration with modern means of communication and innovative forms. Chinese painting culture should be better spread by virtue of modern means of communication through integration with various forms of modern design innovation, to create a good social atmosphere for the inheritance of Chinese painting culture. In international communication, we should respect cultural differences, improve communication methods, and spread Chinese painting culture in a variety of ways. In the international dissemination of Chinese painting culture, we should first master foreigners' understanding of traditional Chinese painting culture. Understanding and mastering the concepts and thinking of foreigners can help better guide them to appreciate Chinese painting. I strongly advocate and practice the cultural exchange between China and the West. As an artist, I have participated in and planned many Chinese and Western joint art exhibitions. By 2018, I have held 6 personal art exhibitions, including 3 times held in the national level museum. The National Art Museum of Kazakhstan has permanently collected my 
three traditional Chinese paintings of Spring Breeze, Ink Bamboo and Beautiful Autumn. In 2018, my personal painting exhibition was held in the National Art Museum of Pavlodar, Kazakhstan, and my works were collected by the museum. My works have also won numerous national and international awards. In 2018, my works Bamboo and Form were selected into the Polish International Art Competition, and my Ink Bamboo was selected into the Contemporary Chinese Ink Bamboo Exhibition. All these are record of my continuous efforts in promoting cultural exchanges between China and the West. I hope that in the spread of Chinese painting, the traditional Chinese philosophy and the spirit of Chinese painting culture can be infiltrated into the heart of the viewers.

\section{Conclusion}

Chinese painting culture has a long history and boasts extensive and profound features. While it faces various problems in inheritance and dissemination in the new era. To this end, we should strengthen the awareness of inheriting Chinese traditional culture and create the appreciation and learning atmosphere of Chinese painting from schools, society and other aspects. Moreover, the innovation of Chinese painting elements should be applied to all aspects of daily life, in order to set off a wave of learning Chinese painting. We should further familiarize with, understand, explore, and study the formal factors of ink paintings, maintain the oriental characteristic and nationality of ink paintings, and imbue the beauty in form of ink painting on the curtain of international art. In this way, Chinese traditional art can be transmitted through successive generations, and the outstanding essence of Chinese painting can be permanently preserved. For this purpose, I am willing to devote my life to the inheritance of China's excellent culture.

\section{References:}

Cao Tiansheng (2017). The unimaginable "past and present" of Xuan paper. Journal of Talent Resources Development, 23, 88-89.

Cao Tiansheng, Su Cheng'ai. (2016). A preliminary study on the concept of "Xuan paper". Journal of Beijing Institute of Graphic Communication, 6, 1.

Chen Chiyu (2016). The essence of Chinese painting: shape expression and spontaneous expression. Art News of China.

Chen Yuxing (2016). Research on the inheritance, transmission and innovation of Chinese painting. Social Sciences in Guangxi, 12, 209-211. 
Chen Zhiwei, XIE Chongkai (1986). Chinese painting and Xuan paper. Paper and Paper Making, 4, 24-25.

Gu Yunfang (2018). Have you chosen the right Xuan paper? Guangdong Print, 4, 4445.

Liu Jieyan, Chang Hong (2018). Zhang Biyun's “Belt and Road” painting exhibition was held in Kazakhstan. Peoples Network, 2.

Liu Renqing (2008). Thinking on some problems in the research and application of Xuan paper. China Pulp \& Paper Industry, 29 (7), 56.

Lu Qing (2018). Research on production process innovation of traditional Xuan paper. China National Exbibition, 2, 20-21.

Mao Wanbao, Huang Jun (2009). Ancient Chinese calligraphy theories. Hefei: Anhui Education Press.

Sun Chunli (2018). Discussion on the material and artistic expression with Chinese meaning. Popular Literature and Art, 22, 128.

Wu Shixin (2013). The production process of Chinese Xuan paper's raw materials. China Pulp \& Paper Industry, 2, 85.

Xia Kejun (2017). Magnificent volume ink. Oriental Art, 19, 104-109.

Yang Ermin (2018). The mystery of color interpreting the color of Chinese painting. Collections, 4, 110-113, 175.

Yin Xing (2017). Ink rhyme - The aesthetic way of Chinese painting's ink color. Beauty and Times, 12, 2, 65-67.

Yuan Chifeng (2018). Discussion on the formal beauty of Chinese painting. Beauty and Times, 3, 2, 57-59.

Zhang Jianzhou, Liang Luning (2018). Historical study on common straw fiber and phloem fiber in the identification of "paper age". Chinese Journal of Forensic Sciences, 2, 22-29.

Zheng Yihao (2017). Discussion on the sentiment of brush and ink in Chinese flower and bird paintings. Art Education Research, 24, 16. 